Journal for ImmunoTherapy of Cancer

\title{
Association between body mass index, dosing strategy, and efficacy of immune checkpoint inhibitors
}

\author{
Murtaza Ahmed (D , ${ }^{1}$ Mitchell S von Itzstein, ${ }^{2}$ Thomas Sheffield, ${ }^{3}$ Shaheen Khan, ${ }^{4}$ \\ Farjana Fattah, ${ }^{5}$ Jason Y Park, ${ }^{4}$ Vinita Popat, ${ }^{1}$ Jessica M Saltarski, ${ }^{5}$ \\ Yvonne Gloria-McCutchen, ${ }^{5}$ David Hsiehchen, ${ }^{2,5}$ Jared Ostmeyer (i) , 3 \\ Saad A Khan, ${ }^{2}$ Nazima Sultana, ${ }^{5}$ Yang Xie, ${ }^{3,5}$ Quan-Zhen Li, ${ }^{6}$ Edward K Wakeland, ${ }^{6}$ \\ David E Gerber $2,3,5$
}

To cite: Ahmed M, von Itzstein MS, Sheffield T, et al. Association between body mass index, dosing strategy, and efficacy of immune checkpoint inhibitors. Journal for ImmunoTherapy of Cancer 2021;9:e002349. doi:10.1136/ jitc-2021-002349

- Additional supplemental material is published online only. To view, please visit the journal online (http://dx.doi.org/10. 1136/jitc-2021-002349).

MA and MSvl contributed equally.

Presented in abstract form at the 2020 ECOG-ACRIN Young Investigator Symposium on September 10, 2020.

Accepted 25 April 2021

\section{Check for updates}

(C) Author(s) (or their employer(s)) 2021. Re-use permitted under CC BY-NC. No commercial re-use. See rights and permissions. Published by BMJ.

For numbered affiliations see end of article.

\section{Correspondence to} Dr David E Gerber; David.Gerber@utsouthwestern. edu

\section{ABSTRACT}

Background Increased body mass index (BMI) has been associated with improved response to immune checkpoint inhibitors (ICls) in multiple cancer types. We evaluated associations between $\mathrm{BMI}, \mathrm{ICl}$ dosing strategy, and clinical outcomes.

Methods We abstracted clinical data on patients with cancer treated with ICI, including age, sex, cancer type, BMI, ICl type, dosing strategy (weight-based or fixed), radiographic response, overall survival (OS), and progression-free survival (PFS). We compared clinical outcomes between low-BMl and high-BMI populations using Kaplan-Meier curves, Cox regressions, and Pearson product-moment correlation coefficients.

Results A total of 297 patients were enrolled, of whom $40 \%$ were women and $59 \%$ were overweight $(\mathrm{BMl} \geq 25)$. Of these, $204(69 \%)$ received fixed and $93(31 \%)$ received weight-based ICI dosing. In the overall cohort, overweight BMI was associated with improved PFS (HR 0.69; $95 \% \mathrm{Cl} 0.51$ to $0.94 ; \mathrm{p}=0.02)$ and had a trend toward improved OS (HR 0.77; $95 \% \mathrm{Cl} 0.57$ to $1.04 ; \mathrm{p}=0.08$ ). For both endpoints, improved outcomes in the overweight population were limited to patients who received weightbased ICI dosing (PFS HR 0.53; $p=0.04$ for weight-based; vs HR $0.79 ; p=0.2$ for fixed dosing) (OS HR $0.56 ; p=0.03$ for weight-based; vs HR 0.89 ; $p=0.54$ for fixed dosing). In multivariable analysis, BMI was not associated with PFS or OS. However, the interaction of $\mathrm{BMI} \geq 25$ and weight-based dosing had a trend toward association with PFS (HR 0.53; $95 \% \mathrm{Cl} 0.26$ to $1.10 ; p=0.09$ ) and was associated with OS (HR 0.50; 95\% Cl 0.25 to 0.99; $p=0.05$ ). Patients with $\mathrm{BMl}<25$ tended to have better outcomes with fixed-dose compared with weight-based ICl, while patients with $\mathrm{BMI} \geq 25$ tended to have better outcomes with weightbased $\mathrm{ICl}$, although these differences did not achieve statistical significance. There was no association between radiographic response and $\mathrm{BMI}$ with fixed-dose $\mathrm{ICI}$ $(p=0.97)$, but a near-significant trend with weight-based $\mathrm{ICI}(p=0.1)$. In subset analyses, the association between $\mathrm{BMI}, \mathrm{ICI}$ dosing strategy, and clinical outcomes appeared limited to men.

Conclusions The clinical benefit of ICl in high-BMI populations appears limited to individuals receiving weight-based ICI dosing. Further research into optimal ICI dosing strategies may be warranted.
In recent years, immune checkpoint inhibitors (ICI) have markedly transformed the treatment of advanced cancers. Although these therapies have led to improved outcomes for a subset of patients, a substantial proportion of patients do not receive benefit. Accordingly, the identification of individuals most likely to benefit from these costly and potentially toxic therapies represents a major area of investigation. To date, the most established predictive markers reflect tumor biology, including programmed death 1 ligand (PDL1) expression, microsatellite instability, and mutational burden. ${ }^{1-5}$

Additionally, certain clinical characteristics are associated with efficacy of ICI. Exposure to steroids has been linked to inferior outcomes, although the extent to which this observation reflects direct immunosuppressive effects versus an indicator of poor prognosis remains unclear. ${ }^{67}$ Antibiotic exposure may also portend reduced benefit from ICI, an association attributed to effects on the gut microbiome. $^{8-10}$ In lung cancer, past or current smoking is associated with high tumor mutational burden, which in turn confers benefit from ICI. ${ }^{411}$

Body mass index (BMI) has also been associated with ICI efficacy, with overweight and obese patients having the best outcomes. ${ }^{12}$ Such observations stand in contrast to the longstanding and predominant view that obesity promotes tumor development and progression, thereby conveying worse prognosis in oncology populations. ${ }^{13}$ In studies in melanoma, lung cancer, and kidney cancer, overweight and/or obese patients receiving immunotherapy have superior outcomes compared with individuals with lower BMI. ${ }^{14-17}$ Conversely, BMI-associated benefits are not observed with conventional 
chemotherapy. ${ }^{14}$ Potential hypotheses to explain this 'obesity paradox' include programmed death-1 (PD-1)driven leptin-mediated dysfunction, adiposity-associated inflammatory cytokines, differences in levels of glutamine and other nutrients essential for immune cell function, increased numbers of proinflammatory primed immune cells (eg, M1 macrophages, CD8+ Tcells) that secrete proinflammatory cytokines (eg, interleukin (IL)-1 $\beta$, IL-6, interferon- $\gamma$ ), classification of previously obese individuals as normal weight due to cancer-associated weight loss, and less aggressive disease among obese individuals. ${ }^{18-23}$

ICI dosing approaches differ across agents and have changed over time. While initially these therapies were primarily dosed according to patient weight, more recently a number of commonly used ICI have adopted fixed-dose regimens. We therefore analyzed dosing strategy, BMI, and outcomes in a cohort of patients with cancer treated with ICI.

\section{METHODS}

\section{Patient selection and study procedures}

This study was conducted within a prospective registry of cancer immunotherapy approved by the UT Southwestern Institutional Review Board (IRB \#STU 082015-053). We identified patients with a confirmed cancer diagnosis who initiated ICI therapy (PD1, PD-L1 and CTLA4 inhibitors) for active disease between November 2015 (registry initiation) and December 2019 at UT Southwestern Medical Center. Other key inclusion criteria included no prior treatment with ICI therapy and availability of serial radiographic studies to assess response.

\section{Clinical data collection and characterization}

For enrolled subjects, we collected the following data: BMI; ICI dosing strategy (weight-based or fixed); age; sex; race/ethnicity; cancer type; type and dates of ICI therapy; type of concurrent/sequential therapy; radiographic response (using Response Evaluation Criteria in Solid Tumors (RECIST) V.1.1. ${ }^{24}$ For BMI determination, we obtained patient weight and height on the day of ICI initiation from the electronic health record. We calculated BMI as weight (in kilograms) divided by the square root of height (in meters). Based on BMI distribution across the study population and consistent with prior studies, we dichotomized BMI as $<25$ and $\geq 25$ (threshold for WHO designation of 'overweight'). ${ }^{14}$ We categorized ICI dosing strategy according to initial ICI treatment. That is, if a patient started treatment with weight-based dosing and then changed to fixed dosing, we considered the case as weight-based dosing. We selected this approach because initial ICI therapy drives treatment outcomes, with treatment continued only if early evaluation suggests efficacy and tolerability. In cases where patients may have received ICI combinations featuring concurrent administration of both weight-based (generally anti-cytotoxic $\mathrm{T}$ lymphocyte antigen 4 (CTLA4) therapy) and fixed-dose ICI (generally anti-PD1/PDL1), we categorized the case as fixed dose because sensitivity analyses excluded CTLA4 therapy. Palliative radiation therapy for control of cancerrelated symptoms or complications was not considered concurrent or sequential therapy. For efficacy assessments, we used the most recent available cross-sectional imaging study (most commonly CT) before ICI initiation as a baseline.

\section{Statistical analysis}

Progression-free survival (PFS) times were computed from the date of ICI initiation to the date of radiographic or clinical progression (assessed by treating clinician) or death, or censored at last known evaluation. Overall survival (OS) was computed from ICI initiation to date of death or censored at last known contact. Best radiographic response was computed as the percent change between the smallest measured sum of tumor diameters after baseline and the sum of diameters at baseline. The fixed dose equivalent for each patient was computed by dividing their total administered dose/week (accounting for weight, where applicable) by the standard fixed dose/ week regimen for the primary ICI agent (anti-PD1/ PDL1 in combinations with anti-CTLA4). A fixed dose equivalent of 1 corresponded to a standard fixed-dose regimen. We computed this value only for patients whose primary ICI agent had both weight-based and fixed-dose regimens during the study: durvalumab, nivolumab, and pembrolizumab.

Kaplan-Meier curves and Cox regressions (including associated statistics) were generated using the $\mathrm{R}$ survival package (V.3.1-8). P values between survival curves were computed using the log-rank test. $\mathrm{P}$ values for box and whisker plots and for best radiographic response were generated using a Mann-Whitney $\mathrm{U}$ test to compare patients with $\mathrm{BMI}<25$ and $\mathrm{BMI} \geq 25$ in the same category. $\mathrm{P}$ values in table 1 were generated by comparing the $B M I<25$ and $B M I \geq 25$ groups using Fisher's exact test for categorical variables and t-tests for continuous variables. All computation was performed using R (V.3.6.3).

\section{RESULTS \\ Patient characteristics}

A total of 297 patients were included in this study. Median age was 68 years, and $120(40 \%)$ were women. Additional case characteristics are shown in table 1. For the 82 cases (27\%) designated as 'other' cancer type, specific diagnoses were as follows: renal cell carcinoma $(n=20)$, head and neck squamous cell carcinoma $(n=21)$, small cell lung cancer $(n=13)$, mesothelioma $(n=4)$, pancreatic cancer $(n=4)$, rectal cancer $(n=3)$, urothelial cancer $(n=3)$, hepatocellular carcinoma $(n=1)$, soft tissue sarcoma $(n=1)$, brain cancer $(n=1)$, ovarian cancer $(\mathrm{n}=1)$, cervical cancer $(\mathrm{n}=1)$, uterine cancer $(\mathrm{n}=1)$, breast cancer $(\mathrm{n}=1)$, non-Hodgkin's lymphoma $(\mathrm{n}=1)$, thyroid cancer $(n=1)$, skin squamous cancer $(n=1)$, cholangiocarcinoma $(\mathrm{n}=1)$, adenoid cystic carcinoma $(\mathrm{n}=1)$, sinonasal cancer $(n=1)$, and unknown primary $(n=1)$. The 78 cases 
Table 1 Case characteristics according to body mass index

\begin{tabular}{|c|c|c|c|c|}
\hline Characteristic & $\begin{array}{l}\text { Total } \\
\text { N (\%) or median (range) }\end{array}$ & $\begin{array}{l}\text { BMI <25 } \\
\text { N (\%) or median (range) }\end{array}$ & $\begin{array}{l}\mathrm{BMI} \geq 25 \\
\mathrm{~N}(\%) \text { or median (range) }\end{array}$ & $P$ value \\
\hline Age (years) & $68(27-92)$ & 68 (27-92) & 67 (33-90) & 0.15 \\
\hline Female & $120(40)$ & $61(50)$ & $59(34)$ & \\
\hline Male & $177(60)$ & $60(50)$ & $117(66)$ & \\
\hline Other & $70(24)$ & $33(27)$ & $37(21)$ & \\
\hline Cancer type & & & & 0.019 \\
\hline $\begin{array}{l}\text { Non-small cell lung } \\
\text { cancer }\end{array}$ & $158(53)$ & $71(59)$ & $87(49)$ & \\
\hline Receipt of anti-CTLA4 & & & & 0.6 \\
\hline Yes & $39(13)$ & $14(12)$ & $25(14)$ & \\
\hline No & $258(87)$ & $107(88)$ & $151(86)$ & \\
\hline Dosing strategy & & & & 0.16 \\
\hline Fixed & $204(69)$ & $89(74)$ & $115(65)$ & \\
\hline Weight-based & $93(31)$ & $32(26)$ & $61(35)$ & \\
\hline
\end{tabular}

BMI, body mass index; CTLA4, cytotoxic T lymphocyte antigen 4.

(26\%) that were classified as concurrent or sequential therapy received the following treatments: concurrent chemotherapy $(n=49)$, concurrent/sequential (chemo) radiation $(n=27)$, concurrent targeted therapy $(n=2)$. Patient BMI was distributed as follows: $\mathrm{BMI}<25 \quad(\mathrm{n}=121$, $41 \%), \quad B M I \geq 25 \quad(n=176,59 \%)$. BMI was significantly associated with gender (men>women) and cancer type (melanoma>other).

Among the enrolled patients, 204 (69\%) received fixed-dose ICI, and $93(31 \%)$ received weight-based ICI. A total of 39 patients $(13 \%)$ received combination ICI (all anti-CTLA4+anti-PD1/PDL1, generally ipilimum$\mathrm{ab}+$ nivolumab). Of these cases, 28 received weight-basing dosing for both drugs. The remaining 12 patients received weight-based ipilimumab plus fixed-dose nivolumab and were characterized as fixed-dose ICI. There was a clear temporal association with dosing approach. The first ICI approved for fixed-dose administration was nivolumab. ${ }^{25}$ Prior to this point, only 1 out of 30 patients $(3 \%)$ in our cohort initiated on ICI received fixed-dose ICI. After this point, 208 out of 269 patients $(77 \%)$ received fixed-dose ICI.

\section{Clinical outcomes}

Median follow-up in the study population was 323 days (IQR 159-575 days). In the overall cohort, patients with higher BMI had improved outcomes with ICI therapy (figure 1). Median PFS was 160 days in the $\mathrm{BMI}<25$ group compared with 305 days for the BMI $\geq 25$ group (HR 0.69; $95 \%$ CI 0.51 to $0.94 ; \mathrm{p}=0.02$ ). Median OS was 414 days in the $\mathrm{BMI}<25$ group compared with 503 days in the $\mathrm{BMI} \geq 25$ group (HR 0.77; 95\% CI 0.57 to $1.04 ; \mathrm{p}=0.08$ ).

Figure 2 displays PFS and OS according to BMI in weightbased and fixed-dose cohorts. With weight-based dosing, overweight patients $(\mathrm{BMI} \geq 25)$ had significantly improved PFS and OS compared with the $\mathrm{BMI}<25$ group. Specifically, median PFS was 81 days for $\mathrm{BMI}<25$ vs 406 days for BMI $\geq 25$ (HR 0.53; 95\% CI 0.3 to 0.96; $\mathrm{p}=0.04$ ). Median OS was 158 days for $\mathrm{BMI}<25$ vs 742 days for $\mathrm{BMI} \geq 25$ (HR $0.56 ; 95 \%$ CI 0.33 to $0.95 ; \mathrm{p}=0.03$ ). By contrast, we observed no difference in outcomes according to BMI with fixed dosing: PFS (HR 0.79; 95\% CI 0.54 to 1.14; $\mathrm{p}=0.2$ ); OS (HR $0.89 ; 95 \%$ CI 0.62 to $1.29 ; \mathrm{p}=0.54$ ).

Cox regression analyses are shown in table 2 . In univariate analysis, higher BMI and melanoma diagnosis had improved PFS. Younger age and melanoma diagnosis had improved OS. While BMI had a significant or nearsignificant association with PFS and OS in univariable analysis, there was no association with either endpoint in multivariable analysis. However, the interaction of BMI and weight-based dosing had a near-significant trend toward association with PFS and was significantly associated with OS.

Because melanoma cases had significantly higher BMI and better clinical outcomes than other cancer types, 

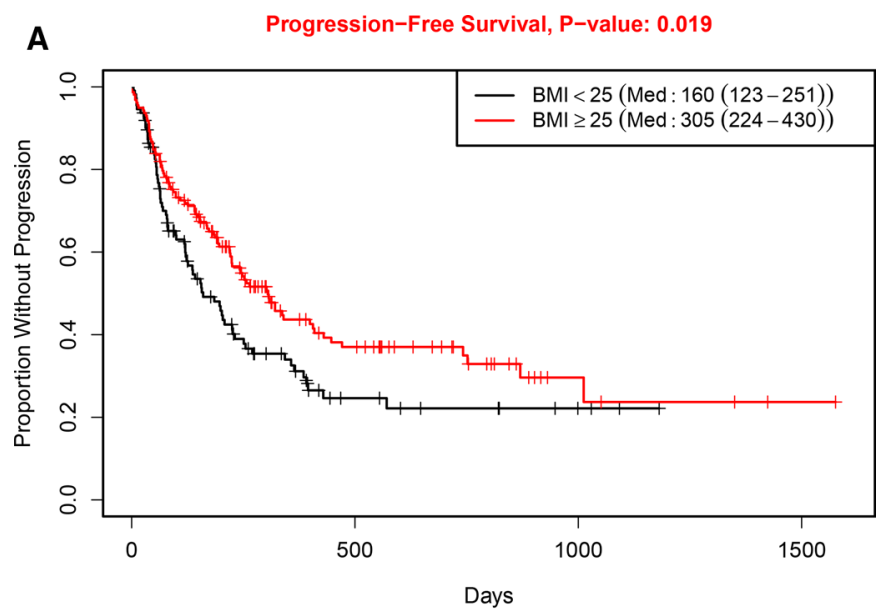

B

Overall Survival, P-value: $\mathbf{0 . 0 8 2}$

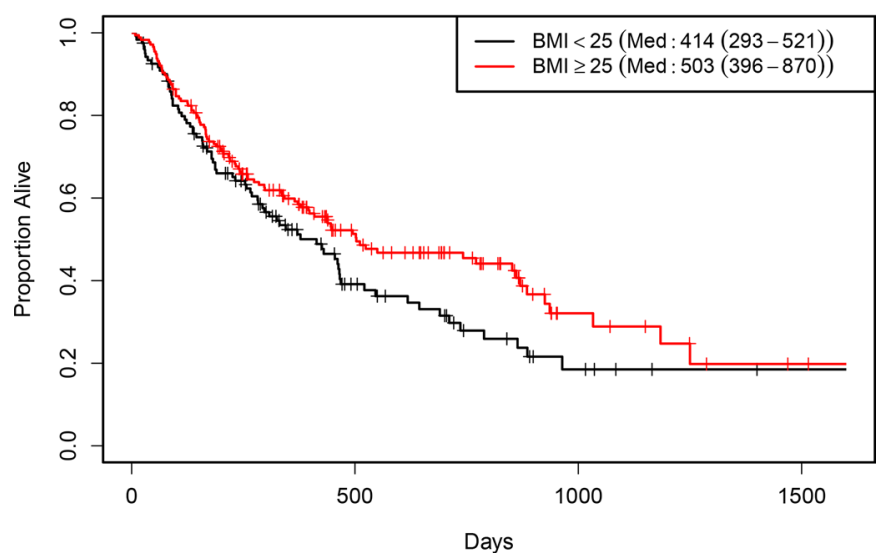

Figure 1 Clinical outcomes according to body mass index (BMI): (A) progression-free survival; (B) overall survival.

we performed the same analyses in the non-melanoma cohort $(\mathrm{n}=240)$ (online supplemental figures 1 and 2). We obtained similar results. The BMI $\geq 25$ group had superior PFS compared with the $\mathrm{BMI}<25$ group with weightbased dosing (HR 0.53; 95\% CI 0.28 to $1.00 ; \mathrm{p}=0.05$ ) but not with fixed dosing (HR 0.82; $95 \%$ CI 0.55 to $1.2 ; \mathrm{p}=0.3$ ). The BMI $\geq 25$ group had nearly significantly superior OS compared with the $\mathrm{BMI}<25$ group with weight-based dosing (HR 0.57; 95\% CI 0.32 to 1.02; $\mathrm{p}=0.06$ ) but not with fixed dosing (HR 1.01; 95\% CI 0.69 to 1.48 ; $\mathrm{p}=0.95$ ).

Similarly, because CTLA4 therapies are most commonly used for melanoma treatment and are only administered by weight-based dosing, we also repeated the analyses after removing cases treated with anti-CTLA4 ICI alone or in combination. In the resulting cohort $(\mathrm{n}=258)$, our findings did not differ meaningfully from the overall study population. $\mathrm{BMI} \geq 25$ had better PFS in the overall cohort $(p=0.02)$ and the weight-based dosing group $(p=0.04)$, but not in the fixed-dose group $(\mathrm{p}=0.2)$. For OS, BMI $\geq 25$ had a trend toward superior outcomes $(p=0.1)$. For weight-based dosing, BMI $\geq 25$ had a trend toward superior OS $(p=0.08)$. However, there was no difference in OS according to BMI with fixed-dose ICI $(\mathrm{p}=0.44)$.

When one considers outcomes according to dosing strategy for each BMI category (online supplemental figure 3), patients with $\mathrm{BMI}<25$ tended to have better outcomes with fixed-dose compared with weight-based ICI (median PFS 199 vs 81 days; $p=0.16$; median OS 455 vs 158 days; $p=0.04)$. Conversely, patients with $\mathrm{BMI} \geq 25 \mathrm{had}$ numerically better outcomes with weight-based compared with fixed-dose ICI, although these differences were not significant (median PFS 406 vs 264 days; $\mathrm{p}=0.39$; median OS 742 vs 502 days; $\mathrm{p}=0.47$ ).

Because underweight BMI has been associated with inferior clinical outcomes in multiple cancer types, we examined whether these patients could be driving our observed results. ${ }^{26-28}$ However, using the WHO definition of $\mathrm{BMI}<18.5$, we identified only nine patients $(3 \%)$ in this category. As would be expected given such small numbers, sensitivity analysis performed after removing these cases demonstrated no meaningful differences from our overall findings (online supplemental figure 4).

Earlier reports have found that the association between BMI and immunotherapy outcomes may be limited to male patients. ${ }^{14}$ Accordingly, we performed our analyses in female-only and male-only cohorts (online supplemental figures 5-7). Overall, in men, we observed a significant difference according to BMI for both PFS ( $\mathrm{p}=0.03$ ) and OS $(p=0.002)$. However, for women, there was no significant difference according to BMI for PFS $(\mathrm{p}=0.2)$ or OS $(p=0.72)$. Among men, there was no significant difference according to BMI with fixed dosing (PFS $\mathrm{p}=0.35$; OS $\mathrm{p}=0.25)$. However, with weight-based dosing, patients with $B M I \geq 25$ had significantly better PFS $(p=0.02)$ and OS $(\mathrm{p}<0.001)$. Among women, there was no difference in PFS or OS according to BMI, regardless of ICI dosing strategy.

Earlier studies have suggested discrete effects of obese (in contrast to overweight) status on BMI outcomes, with some reports identifying further benefit and others noting less advantage. ${ }^{1420}$ In the present study, obese and overweight individuals had similar clinical outcomes (online supplemental figure 8).

Figure 3 displays the best radiographic response measured by RECIST according to BMI in the overall cohort and separate weight-based and fixed-dose cohorts. With weight-based dosing, there was a trend toward a greater reduction in tumor measurements in patients with BMI $\geq 25 \quad(p=0.1)$. With fixed dosing, there was no association between BMI and radiographic response $(p=0.97)$.

To investigate further the association between dosing methods and outcomes, we analyzed fixed-dose equivalents according to BMI in all patients and in weightbased and fixed-dose cohorts (figure 4). In the overall cohort, patients with a $\mathrm{BMI} \geq 25$ had a significantly greater dose equivalent fraction $(p=0.003)$. In the weight-based dosing cohort, the difference in dose exposure was more pronounced and had a near significant trend $(\mathrm{p}=0.08)$.

\section{DISCUSSION}

In recent years, BMI has joined the ranks of smoking history, steroid exposure, antibiotic use, HLA type, and 

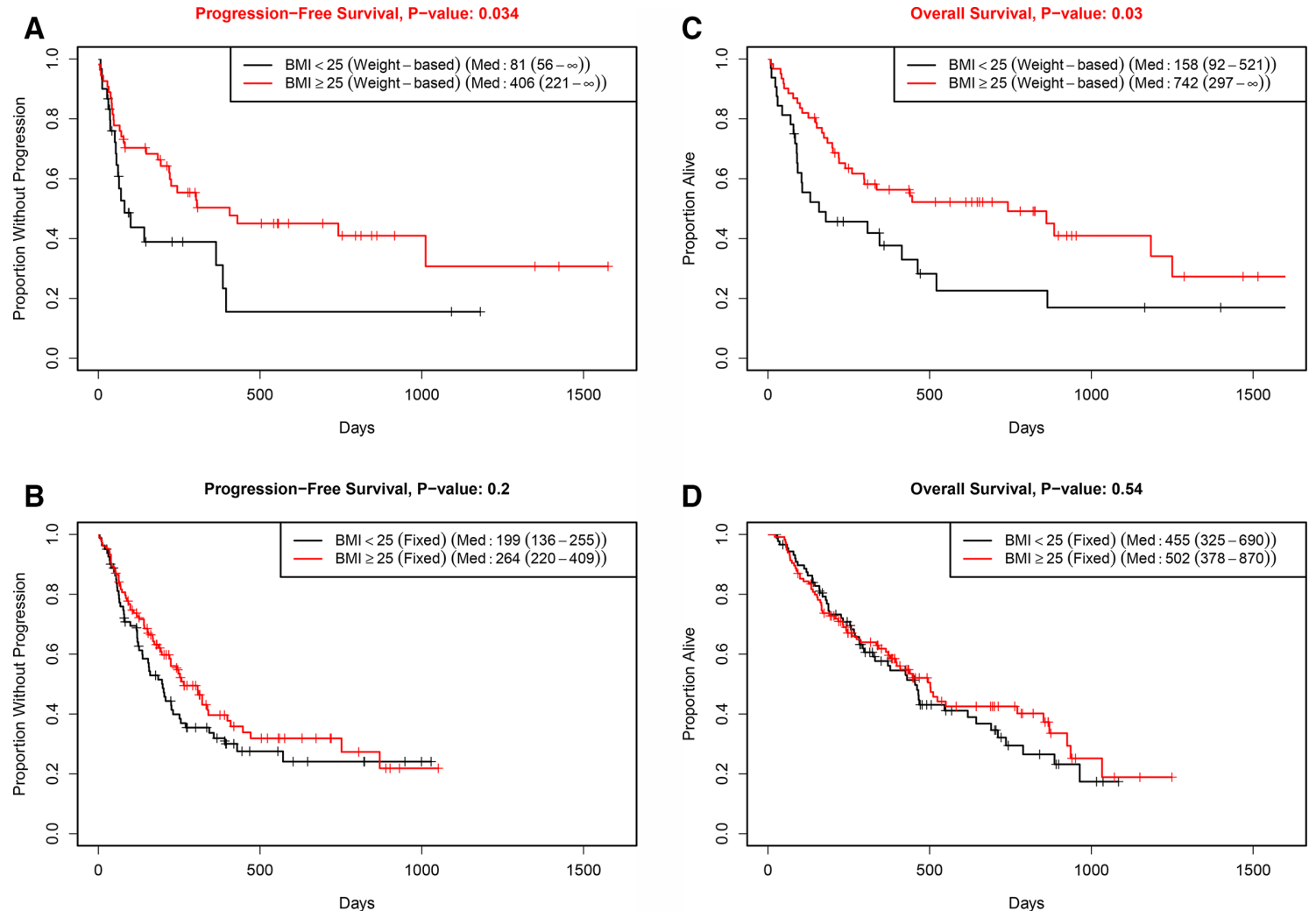

Figure 2 Clinical outcomes according to body mass index (BMI) and dosing strategy. (A) Progression-free survival with weightbased dosing; (B) progression-free survival with fixed dosing; (C) overall survival with weight-based dosing; (D) overall survival with fixed dosing.

tumor characteristics (including PDL1 expression, mutational burden, and microsatellite instability) as a potential predictor of immunotherapy efficacy. Specifically, patients with higher BMI, whether categorized as overweight (BMI 25-29) or obese (BMI $\geq 30$ ) have been shown to have more favorable outcomes from checkpoint inhibitors than patients with lower BMI. ${ }^{12-17}$ In the present study, we examined clinical outcomes not only according to patient BMI, but also according to ICI dosing strategy (which differs among ICI types and has changed over time).

As with prior studies, we identified improved outcomes in overweight patients. However, this benefit appeared limited to those patients who received weight-based ICI and was not apparent in patients who received fixeddose ICI. This benefit spanned all efficacy endpoints, including radiographic response, PFS, and OS. Furthermore, patients with $\mathrm{BMI}<25$ tended to have better outcomes with fixed-dose compared with weight-based ICI, while patients with $\mathrm{BMI} \geq 25$ tended to have better outcomes with weight-based compared with fixed-dose ICI. These observations are all the more noteworthy because the weight-based ICI cohort represented less than one-third of the overall study population, suggesting that the statistically significant differences in outcomes reflect large effect sizes. Removal of outlying subgroups, such as patients with melanoma, patients receiving antiCTLA4 therapy, or underweight patients, did not alter our findings. Additionally, although the use of fixeddose ICI has coincided to some extent with approvals for combination regimens incorporating chemotherapy, targeted therapy, and/or radiation therapy, controlling for receipt of sequential or concurrent therapies did not impact results.

To place these observations in context, it is worth reviewing the evolution of anti-PD1/PDL1 therapy dosing. For the anti-PD1 agent pembrolizumab, clinical trials in melanoma and lung cancer initially employed two weight-based doses-2 and $10 \mathrm{mg} / \mathrm{kg}$ intravenous ${ }^{29}{ }^{30}$ which were found to be equivalent in a lung cancer trial. ${ }^{31}$ Subsequent trials and indications of pembrolizumab used a fixed dose of $200 \mathrm{mg}$ intravenous every 3 weeks, with the approval of $400 \mathrm{mg}$ intravenous every 6 weeks in $2020 .^{32}$ The anti-PD1 agent nivolumab was initially dosed by weight $(3 \mathrm{mg} / \mathrm{kg})$, but since 2016 has been available as fixed dose (240 mg every 2 weeks) and more recently as $480 \mathrm{mg}$ every 4 weeks. ${ }^{25} 3334$

Because most ICI clinical trials did not collect intensive, serial time-course pharmacokinetic samples, the potential application of fixed dosing has been investigated using population pharmacokinetics. ${ }^{33} 35$ For pembrolizumab, after establishing the range of exposures from dose regimens with comparable efficacy and tolerability (ranging from 5th percentile of $2 \mathrm{mg} / \mathrm{kg} \mathrm{q3}$ wks to $95 \mathrm{th}$ percentile of $10 \mathrm{mg} / \mathrm{kg} \mathrm{q} 2 \mathrm{wks}$ ), it was determined that a fixed dose of $200 \mathrm{mg} / \mathrm{kg}$ q3wks would have substantial overlap with 
Table 2 Progression-free and overall survival Cox regression analyses

\begin{tabular}{|c|c|c|c|c|}
\hline \multirow[b]{2}{*}{ Covariate } & \multicolumn{2}{|l|}{ Univariate analysis } & \multicolumn{2}{|c|}{ Multivariate analysis } \\
\hline & HR $(95 \% \mathrm{Cl})$ & $P$ value & HR $(95 \% \mathrm{Cl})$ & $P$ value \\
\hline \multicolumn{5}{|l|}{ Progression-free survival } \\
\hline Age (continuous variable) & 1.01 (1.00 to 1.03$)$ & 0.07 & 1.01 (0.99 to 1.03$)$ & 0.26 \\
\hline Male gender & 1.14 (0.83 to 1.57$)$ & 0.43 & 1.32 (0.94 to 1.86$)$ & 0.11 \\
\hline Non-Hispanic white race/ethnicity & 1.03 (0.71 to 1.50$)$ & 0.88 & 1.11 (0.76 to 1.63$)$ & 0.59 \\
\hline \multicolumn{5}{|l|}{ Cancer type } \\
\hline NSCLC & 1.02 (0.72 to 1.45$)$ & 0.91 & $1.13(0.77$ to 1.66$)$ & 0.54 \\
\hline Melanoma & 0.37 (0.21 to 0.67$)$ & $<0.001$ & 0.39 (0.22 to 0.72$)$ & 0.002 \\
\hline Anti-CTLA4 administered & 1.11 (0.69 to 1.42$)$ & 0.66 & $1.80(1.02$ to 3.19$)$ & 0.04 \\
\hline Concurrent/sequential therapy & 1.24 (0.89 to 1.74$)$ & 0.21 & $1.13(0.79$ to 1.62$)$ & 0.51 \\
\hline $\mathrm{BMI} \geq 25$ & 0.69 (0.51 to 0.94$)$ & 0.02 & 0.87 (0.59 to 1.28$)$ & 0.48 \\
\hline Weight-based dose ICI & 0.96 (0.68 to 1.34$)$ & 0.79 & 1.39 (0.80 to 2.42$)$ & 0.24 \\
\hline $\mathrm{BMI} \geq 25 /$ Weight-based dose interaction & NA & NA & $0.53(0.26$ to 1.10$)$ & 0.09 \\
\hline \multicolumn{5}{|l|}{ Overall survival } \\
\hline Age (continuous variable) & 1.02 (1.01 to 1.04$)$ & 0.004 & $1.02(1.00$ to 1.03$)$ & 0.03 \\
\hline Male gender & 1.19 (0.88 to 1.62$)$ & 0.26 & $1.41(1.01$ to 1.97$)$ & 0.04 \\
\hline Non-Hispanic white race/ethnicity & 1.20 (0.83 to 1.72$)$ & 0.33 & 1.34 (0.92 to 1.97$)$ & 0.13 \\
\hline \multicolumn{5}{|l|}{ Cancer type } \\
\hline NSCLC & 1.26 (0.89 to 1.79$)$ & 0.19 & $1.26(0.86$ to 1.85$)$ & 0.24 \\
\hline Melanoma & 0.47 (0.27 to 0.81$)$ & 0.007 & $0.43(0.24$ to 0.77$)$ & 0.004 \\
\hline Anti-CTLA4 administered & 1.00 (0.64 to 1.57$)$ & 0.99 & 1.70 (1.01 to 2.88$)$ & 0.05 \\
\hline Concurrent/sequential therapy & 1.16 (0.82 to 1.62$)$ & 0.41 & 1.09 (0.76 to 1.57$)$ & 0.64 \\
\hline $\mathrm{BMI} \geq 25$ & 0.77 (0.57 to 1.04$)$ & 0.08 & 0.96 (0.65 to 1.42$)$ & 0.85 \\
\hline Weight-based dose $\mathrm{ICI}$ & 1.04 (0.75 to 1.44$)$ & 0.81 & 1.57 (0.93 to 2.64$)$ & 0.09 \\
\hline $\mathrm{BM} \geq 25 /$ Weight-based dose interaction & NA & NA & 0.5 (0.25 to 0.99$)$ & 0.05 \\
\hline
\end{tabular}

BMI, body mass index; CTLA4, cytotoxic T lymphocyte antigen 4; ICl, immune checkpoint inhibitor; irAE, immune-related adverse event; NSCLC, non-small cell lung cancer.

the $2 \mathrm{mg} / \mathrm{kg}$ q3wks dose. ${ }^{36}$ While fixed-dose nivolumab and pembrolizumab simplifies prescribing, preparation, and inventory, potentially improving safety by reducing dosing errors, a number of studies have projected that fixed-dose results in increased drug costs, translating to a difference of hundreds of thousands of dollars annually across the growing population of patients eligible for ICI. ${ }^{37-39}$ Other concerns include inadequate accounting for the complexity of dose modeling for checkpoint inhibitors (which often include an immune-related biomarker such as IL-2 release as well as translational $\mathrm{PK} / \mathrm{PD}$ response models from preclinical studies) and insufficient power to compare directly outcomes from 2 and $10 \mathrm{mg} / \mathrm{kg}$ pembrolizumab dose cohorts in clinical trials. ${ }^{40}$ It is also possible that $10 \mathrm{mg} / \mathrm{kg}$ may not be an ideal comparator, as it has been suggested that high-dose antibody administration paradoxically results in reduced exposure. Potential explanations include saturable endocytosis and/or saturable degradation processes, ${ }^{41}$ such as the neonatal Fc receptor recycling process, ${ }^{42}{ }^{43}$ thereby leading to increased antibody clearance and reduced half-life.

How should we interpret our results against findings from earlier studies identifying associations between elevated BMI and favorable ICI outcomes? It seems likely that patients included in these other studies primarily or exclusively received weight-based ICI dosing. A large Italian registry study of nivolumab for kidney cancer included patients treated July 2015 through April 2016, which precedes the late 2016 approval of fixed-dose nivolumab. ${ }^{16}$ Similarly, a study published in February 2018 of patients with melanoma treated with various PD1/PDL1 inhibitors had median cohort follow-up of 25 months, suggesting that most patients would have initiated nivolumab or pembrolizumab prior to the approval of fixed-dose regimens. ${ }^{14}$

It is also important to consider the impact of patient size on the pharmacokinetic impact of fixed-dose approaches. In the present study, in the overweight population, we observed a benefit of weight-based dosing. We 

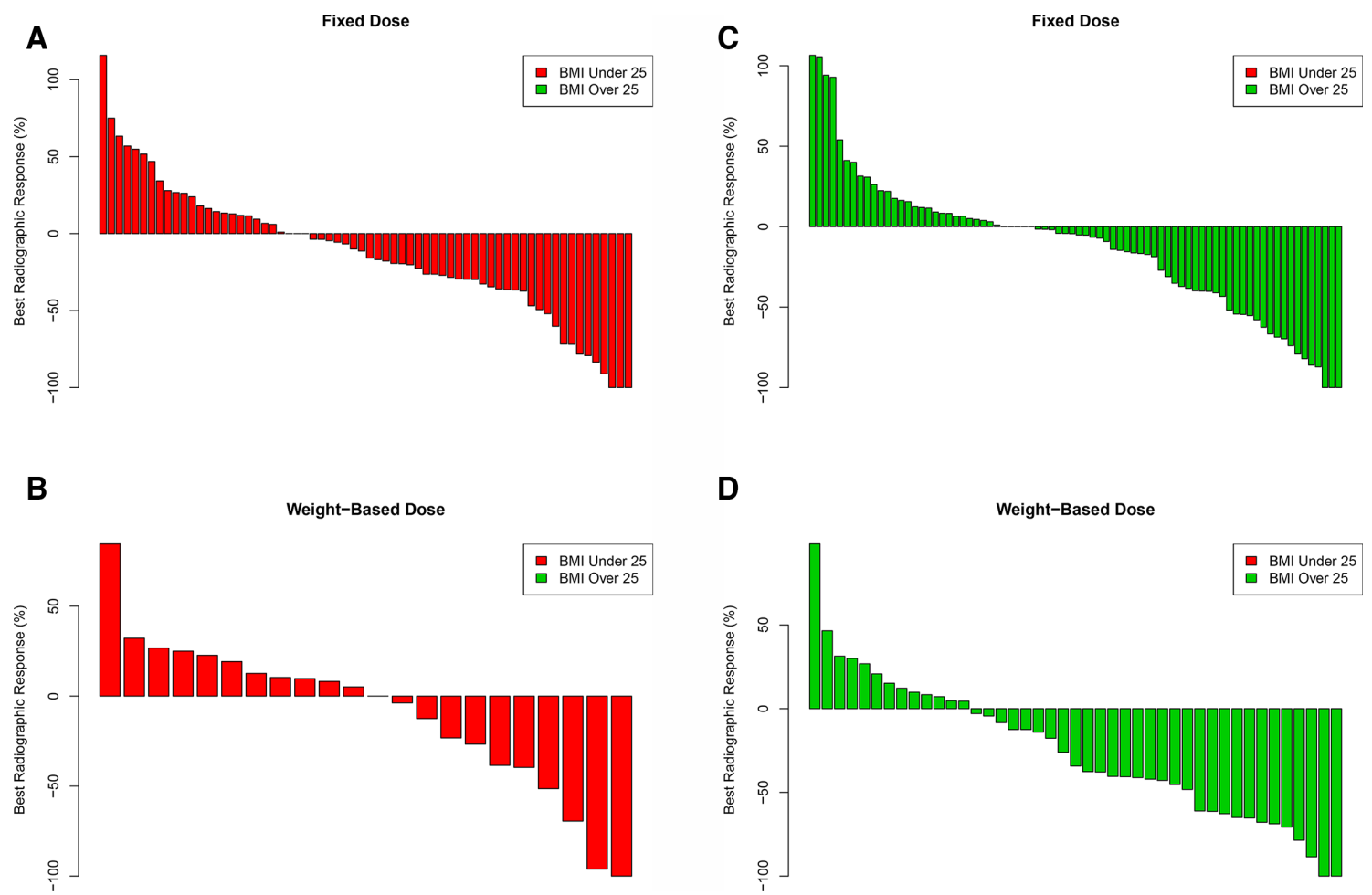

Figure 3 Waterfall plots comparing best radiographic response according to body mass index (BMI) in weight-based and fixed-dose cohorts. (A) Fixed-dose, BMI<25; (B) weight-based dosing, BMI<25; (C) fixed dose, BMI $\geq 25$; (D) weight-based dosing, $\mathrm{BMI} \geq 25$.

further observed that in patients treated with weightbased dosing, overweight and obese patients receive relatively higher dose than patients with $\mathrm{BMI}<25$. However, in a population study of 273 Japanese patients, fixed-dose nivolumab $240 \mathrm{mg}$ intravenous every 2 weeks led to a $37 \%$ increase in exposure compared with $3 \mathrm{mg} / \mathrm{kg}$ intravenous every 2 weeks. ${ }^{44}$ This finding reflects expected regional patient characteristics, as the average adult weight in Asia is $58 \mathrm{~kg}$, and only $24 \%$ of individuals are overweight. ${ }^{45}$ By contrast, in North America, where over $40 \%$ of metastatic cancers may be eligible for ICI therapy, ${ }^{46}$ the average adult weight is $81 \mathrm{~kg}$, with $74 \%$ overweight. ${ }^{45}$

Consistent with earlier studies, we found that the association between BMI and ICI clinical outcomes appeared limited to male patients. ${ }^{1420}$ Potential explanations for

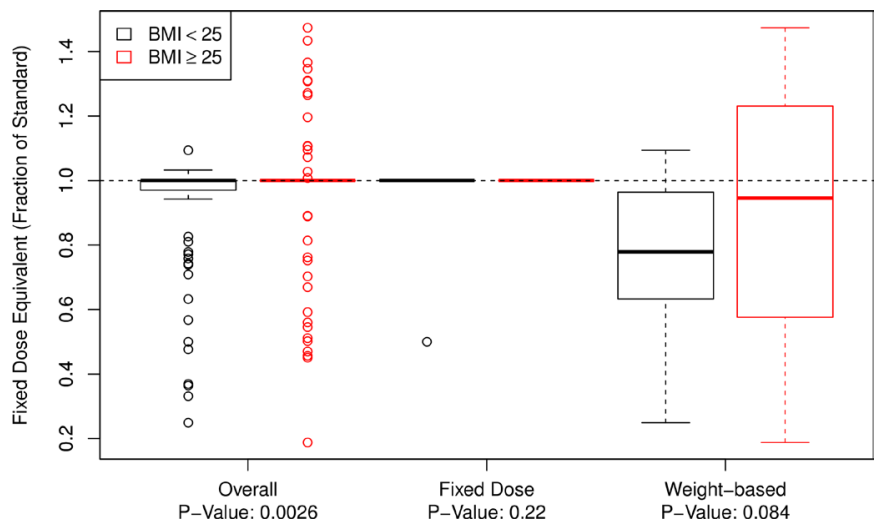

Figure 4 Fixed-dose equivalents according to body mass index (BMI) in weight-based and fixed-dose cohorts. this observation include differences in muscle mass, as skeletal muscle supplies essential nutrients, such as glutamine, for lymphocyte and monocyte function. ${ }^{47}$ With other studies not confirming this finding, there is clearly a need for more investigation in this area. ${ }^{49}$

Key strengths of this study include the detailed clinical data abstraction and ample clinical follow-up. Limitations include the absence of tumor-related predictive markers and clinical information potentially relevant to ICI efficacy such as steroid use, antibiotic exposure, and smoking history, as well as performance status. We also recognize that characteristics of a single-institution patient cohort may not be generalizable across centers. The proportion of overweight patients was somewhat lower than that for the general US adult population ( $59 \%$ vs $74 \%$ ), which could reflect the older age or nutritional status of individuals with cancer. Fewer than $5 \%$ of cases were underweight, preventing analysis of a population associated with adverse clinical outcomes in multiple cancer types, and a relatively small sample size may have obscured differences between overweight and obese patients. ${ }^{26-28}$ Finally, the present study does not provide mechanistic insight into these novel clinical observations.

In conclusion, as noted previously, this study found that overweight patients appear to experience superior outcomes from cancer immunotherapy. However, this clinical benefit may be limited to weight-based ICI. Because the prevalence of overweight and obesity is increasing in the USA and globally, and the most commonly used ICI now employ fixed-dosing approaches, further research 
into the interplay between patient characteristics, ICI dosing strategy, and treatment efficacy are warranted.

\section{Author affiliations}

${ }^{1}$ School of Medicine, The University of Texas Southwestern Medical Center, Dallas,

Texas, USA

${ }^{2}$ Department of Internal Medicine (Division of Hematology-Oncology), The University of Texas Southwestern Medical Center, Dallas, Texas, USA

${ }^{3}$ Department of Population and Data Sciences, The University of Texas Southwestern Medical Center, Dallas, Texas, USA

${ }^{4}$ Department of Pathology, University of Texas Southwestern Medical Center, Dallas, Texas, USA

${ }^{5}$ Harold C. Simmons Comprehensive Cancer Center, The University of Texas

Southwestern Medical Center, Dallas, Texas, USA

${ }^{6}$ Department of Immunology, University of Texas Southwestern Medical Center at Dallas, Dallas, Texas, USA

Present affiliations The present affiliation of Saad A Khan is: Stanford University, California, USA.

\section{Twitter Murtaza Ahmed @murtaza.ahm}

Acknowledgements The authors thank Ms Dru Gray for assistance with manuscript preparation.

Contributors Conception and design: MA, MSvI, DEG. Acquisition of data: MA, MSvl, VP, NS, YG-M. Analysis and interpretation: TS, YX, MA, MSvl, DEG, JO, Q-ZL, EKW, SAK, SK, FF. Drafting of manuscript: MA, MSvl, DEG, TS. Critical revision: All authors.

Funding Funded in part by a National Cancer Institute Midcareer Investigator Award in Patient-Oriented Research (K24 CA201543-01; to DEG), the National Institute of Allergy and Infectious Disease (1U01Al156189-01; to DEG, EKW, YX), an American Cancer Society-Melanoma Research Alliance Team Award (MRAT-18-11401-LIB; to DEG), a V Foundation Robin Roberts Cancer Survivorship Award (DT2019007; to DEG), the University of Texas Lung Cancer Specialized Program of Research Excellence (SPORE) (P50CA070907-21), and the Harold C. Simmons Comprehensive Cancer Center Data Sciences Shared Resource (1P30 CA 142543-03).

Competing interests Drs SAK, SK, FF, JYP, YX, QZ-L, EKW, and DEG report a US patent application $(62 / 654,025)$.

Patient consent for publication Not required.

Provenance and peer review Not commissioned; externally peer reviewed.

Data availability statement Data are available on reasonable request. The dataset using during the present study, including deidentified participant data, is available from the corresponding author on reasonable request.

Supplemental material This content has been supplied by the author(s). It has not been vetted by BMJ Publishing Group Limited (BMJ) and may not have been peer-reviewed. Any opinions or recommendations discussed are solely those of the author(s) and are not endorsed by BMJ. BMJ disclaims all liability and responsibility arising from any reliance placed on the content. Where the content includes any translated material, BMJ does not warrant the accuracy and reliability of the translations (including but not limited to local regulations, clinical guidelines, terminology, drug names and drug dosages), and is not responsible for any error and/or omissions arising from translation and adaptation or otherwise.

Open access This is an open access article distributed in accordance with the Creative Commons Attribution Non Commercial (CC BY-NC 4.0) license, which permits others to distribute, remix, adapt, build upon this work non-commercially, and license their derivative works on different terms, provided the original work is properly cited, appropriate credit is given, any changes made indicated, and the use is non-commercial. See http://creativecommons.org/licenses/by-nc/4.0/.

\section{ORCID iDs}

Murtaza Ahmed http://orcid.org/0000-0002-2964-1069 Jared Ostmeyer http://orcid.org/0000-0003-0405-6987

\section{REFERENCES}

1 Topalian SL, Taube JM, Anders RA, et al. Mechanism-driven biomarkers to guide immune checkpoint blockade in cancer therapy. Nat Rev Cancer 2016;16:275-87.
2 Overman MJ, Lonardi S, Wong KYM, et al. Durable clinical benefit with nivolumab plus ipilimumab in DNA mismatch Repair-Deficient/ Microsatellite Instability-High metastatic colorectal cancer. J Clin Oncol 2018:36:773-9.

3 Yarchoan M, Johnson BA, Lutz ER, et al. Targeting neoantigens to augment antitumour immunity. Nat Rev Cancer 2017;17:209-22.

4 Rizvi NA, Hellmann MD, Snyder A, et al. Cancer immunology. Mutational landscape determines sensitivity to PD-1 blockade in non-small cell lung cancer. Science 2015;348:124-8.

5 Yarchoan M, Hopkins A, Jaffee EM. Tumor mutational burden and response rate to PD-1 inhibition. N Engl J Med 2017;377:2500-1.

6 Arbour KC, Mezquita L, Long N, et al. Impact of baseline steroids on efficacy of programmed cell death-1 and programmed Death-Ligand 1 blockade in patients with non-small-cell lung cancer. $J$ Clin Oncol 2018;36:2872-8.

7 Ricciuti B, Dahlberg SE, Adeni A, et al. Immune checkpoint inhibitor outcomes for patients with non-small-cell lung cancer receiving baseline corticosteroids for palliative versus Nonpalliative indications. $J$ Clin Oncol 2019;37:1927-34.

8 Pinato DJ, Howlett S, Ottaviani D, et al. Association of prior antibiotic treatment with survival and response to immune checkpoint inhibitor therapy in patients with cancer. JAMA Oncol 2019;5:1774.

9 Gopalakrishnan V, Spencer CN, Nezi L, et al. Gut microbiome modulates response to anti-PD-1 immunotherapy in melanoma patients. Science 2018;359:97-103.

10 Matson V, Fessler J, Bao R, et al. The commensal microbiome is associated with anti-PD-1 efficacy in metastatic melanoma patients. Science 2018;359:104-8.

11 Norum J, Nieder C. Tobacco smoking and cessation and PD-L1 inhibitors in non-small cell lung cancer (NSCLC): a review of the literature. ESMO Open 2018;3:e000406.

12 Murphy WJ, Longo DL. The surprisingly positive association between obesity and cancer immunotherapy efficacy. JAMA 2019;321:1247-8.

13 Renehan AG, Tyson M, Egger M, et al. Body-Mass index and incidence of cancer: a systematic review and meta-analysis of prospective observational studies. Lancet 2008;371:569-78.

14 McQuade JL, Daniel CR, Hess KR, et al. Association of bodymass index and outcomes in patients with metastatic melanoma treated with targeted therapy, immunotherapy, or chemotherapy: a retrospective, multicohort analysis. Lancet Oncol 2018;19:310-22.

15 Zhou J, Zhou F, Chu X, et al. Non-alcoholic fatty liver disease is associated with immune checkpoint inhibitor-based treatment response in patients with non-small cell lung cancer with liver metastases. Transl Lung Cancer Res 2020;9:316-24.

16 De Giorgi U, Procopio G, Giannarelli D, et al. Association of systemic inflammation index and body mass index with survival in patients with renal cell cancer treated with nivolumab. Clin Cancer Res 2019;25:3839-46.

17 Ichihara E, Harada D, Inoue K, et al. The impact of body mass index on the efficacy of anti-PD-1/PD-L1 antibodies in patients with nonsmall cell lung cancer. Lung Cancer 2020;139:140-5.

18 Wang Z, Aguilar EG, Luna Jl, et al. Paradoxical effects of obesity on T cell function during tumor progression and PD-1 checkpoint blockade. Nat Med 2019;25:141-51.

19 Mirsoian A, Bouchlaka MN, Sckisel GD, et al. Adiposity induces lethal cytokine storm after systemic administration of stimulatory immunotherapy regimens in aged mice. J Exp Med 2014;211:2373-83.

20 Naik GS, Waikar SS, Johnson AEW, et al. Complex inter-relationship of body mass index, gender and serum creatinine on survival: exploring the obesity paradox in melanoma patients treated with checkpoint inhibition. J Immunother Cancer 2019;7:89.

21 Kane H, Lynch L. Innate immune control of adipose tissue homeostasis. Trends Immunol 2019;40:857-72.

22 Lennon $\mathrm{H}$, Sperrin $\mathrm{M}$, Badrick $\mathrm{E}$, et al. The obesity paradox in cancer: a review. Curr Oncol Rep 2016:18:56.

23 Hakimi AA, Furberg H, Zabor EC, et al. An epidemiologic and genomic investigation into the obesity paradox in renal cell carcinoma. J Natl Cancer Inst 2013;105:1862-70.

24 Eisenhauer EA, Therasse P, Bogaerts J, et al. New response evaluation criteria in solid tumours: revised RECIST guideline (version 1.1). Eur J Cancer 2009;45:228-47.

25 US Food and Drug Administration. Modification of the dosage regimen for nivolumab. Available: https://www.fda.gov/drugs/ resources-information-approved-drugs/modification-dosageregimen-nivolumab [Accessed 31 Aug 2020].

26 Jiang M, Fares AF, Shepshelovich D, et al. The relationship between body-mass index and overall survival in non-small cell lung cancer by sex, smoking status, and race: a pooled analysis of 20,937 international lung cancer Consortium (ILCCO) patients. Lung Cancer 2021;152:58-65. 
27 Fukumoto K, Mori S, Shintani Y, et al. Impact of the preoperative body mass index on the postoperative outcomes in patients with completely resected non-small cell lung cancer: a retrospective analysis of 16,503 cases in a Japanese lung cancer registry study. Lung Cancer 2020;149:120-9.

$28 \mathrm{Kim} \mathrm{LH}$, Doan $\mathrm{P}, \mathrm{He} \mathrm{Y}$, et al. A systematic review and meta-analysis of the significance of body mass index on kidney cancer outcomes. $J$ Urol 2021;205:346-55.

29 Garon EB, Rizvi NA, Hui R, et al. Pembrolizumab for the treatment of non-small-cell lung cancer. N Engl J Med 2015;372:2018-28.

30 Herbst RS, Baas P, Kim D-W, et al. Pembrolizumab versus docetaxel for previously treated, PD-L1-positive, advanced non-small-cell lung cancer (KEYNOTE-010): a randomised controlled trial. Lancet 2016;387:1540-50.

31 Reck M, Rodríguez-Abreu D, Robinson AG, et al. Pembrolizumab versus chemotherapy for PD-L1-positive non-small-cell lung cancer. N Engl J Med 2016;375:1823-33.

32 US Food and Drug Administration. FDA approves new dosing regimen for pembrolizumab. Available: https://www.fda.gov/drugs/ drug-approvals-and-databases/fda-approves-new-dosing-regimenpembrolizumab [Accessed 31 Aug 2020].

33 Zhao X, Suryawanshi S, Hruska M, et al. Assessment of nivolumab benefit-risk profile of a $240-\mathrm{mg}$ flat dose relative to a $3-\mathrm{mg} / \mathrm{kg}$ dosing regimen in patients with advanced tumors. Ann Oncol 2017;28:2002-8.

34 Long GV, Tykodi SS, Schneider JG, et al. Assessment of nivolumab exposure and clinical safety of $480 \mathrm{mg}$ every 4 weeks flat-dosing schedule in patients with cancer. Ann Oncol 2018;29:2208-13.

35 Ahamadi M, Freshwater T, Prohn M, et al. Model-Based characterization of the pharmacokinetics of pembrolizumab: a humanized anti-PD-1 monoclonal antibody in advanced solid tumors. CPT Pharmacometrics Syst Pharmacol 2017;6:49-57.

36 Freshwater T, Kondic A, Ahamadi M, et al. Evaluation of dosing strategy for pembrolizumab for oncology indications. J Immunother Cancer 2017;5:43

37 Mukherjee S, Ibrahimi S, Machiorlatti M, et al. Personalized dosing versus fixed dosing of immune checkpoint inhibitors: a cost analysis study. Am J Ther 2018;25:e767-8.
38 Bianconi C, Gandini G, Zanotti G. 3PC-020 Nivolumab weight-based dosing vs flat dose economic analysis. European Journal of Hospital Pharmacy 2019;26:A45-6.

39 Francis S, Hatton R, Sababa S. Cost minimization evaluation of nivolumab dosing strategies: flat dose versus WeightBased with dose capping. Journal of Oncology Pharmacy Practice 2019;25:2.

40 Tu H-Y, Zhang Q, Wu Y-L, et al. Optimal pembrolizumab dosing for non-small cell lung cancer: further studies still needed. J Thorac Dis 2017;9:4821-4.

41 Mortensen DL, Walicke PA, Wang X, et al. Pharmacokinetics and pharmacodynamics of multiple Weekly subcutaneous efalizumab doses in patients with plaque psoriasis. J Clin Pharmacol 2005;45:286-98.

42 Ryman JT, Meibohm B. Pharmacokinetics of monoclonal antibodies. CPT Pharmacometrics Syst Pharmacol 2017;6:576-88.

43 Jin F, Balthasar JP. Mechanisms of intravenous immunoglobulin action in immune thrombocytopenic purpura. Hum Immunol 2005;66:403-10.

44 Bei D, Osawa M, Uemura S, et al. Benefit-risk assessment of nivolumab $240 \mathrm{mg}$ flat dose relative to $3 \mathrm{mg} / \mathrm{kg}$ Q2W regimen in Japanese patients with advanced cancers. Cancer Sci 2020;111:528-35.

45 Walpole SC, Prieto-Merino D, Edwards P, et al. The weight of nations: an estimation of adult human biomass. BMC Public Health 2012;12:439.

46 Haslam A, Prasad V. Estimation of the percentage of US patients with cancer who are eligible for and respond to checkpoint inhibitor immunotherapy drugs. JAMA Netw Open 2019;2:e192535.

47 Juretic A, Spagnoli GC, Hörig H, et al. Glutamine requirements in the generation of lymphokine-activated killer cells. Clin Nutr 1994;13:42-9.

48 Spittler A, Winkler S, Götzinger P, et al. Influence of glutamine on the phenotype and function of human monocytes. Blood 1995;86:1564-9.

$49 \mathrm{Xu} \mathrm{H}, \mathrm{Cao} \mathrm{D}, \mathrm{He} \mathrm{A}$, et al. The prognostic role of obesity is independent of sex in cancer patients treated with immune checkpoint inhibitors: a pooled analysis of 4090 cancer patients. Int Immunopharmacol 2019;74:105745. 